\title{
Experimental Study on Unidirectional Pedestrian Descending and Ascending Stair With a Fixed Obstacle
}

\author{
Shi Dongdong ${ }^{1} \cdot$ Yang Meicheng ${ }^{2} \cdot$ Chen Juan $^{3} \cdot$ Ma Jian ${ }^{1, *}$ \\ ${ }^{1}$ School of Transportation and Logistics, National Engineering Laboratory of Integrated \\ Transportation Big Data Application Technology, Southwest Jiaotong University, Chengdu, \\ China, \\ E-mail: shidongdong@my.swjtu.edu.cn, majian@mail.ustc.edu.cn *corresponding author. \\ ${ }^{2}$ China Railway Eryuan Engineering Group Co.Ltd, Chengdu, China, \\ E-mail: yangmc727@163.com \\ ${ }^{3}$ Department of Fire Safety Engineering, Southwest Jiaotong University, Chengd, China, \\ E-mail: juanchen@swjtu.edu.cn
}

Received: 15 August 2021 / Last revision received: 16 November 2021 / Accepted: 22 November 2021 DOI: $10.17815 / C D .2021 .127$

\begin{abstract}
Staircase is one of the most essential vertical passageway for pedestrians timely evacuation, and has distinct constraint on pedestrians movement characteristics when compared with corridors and hallways. During evacuation, temporary obstacles such as an abruptly stopped pedestrians or the luggage pedestrians discarded can be observed on stairs. It is noticed that studies on the impact of obstacle on the pedestrian movement characteristics on stairs are rare. Therefore, a series of unidirectional pedestrian flow experiments on stairs were performed to explore the obstacle avoidance behavior. The obstacle avoidance characteristic of pedestrians is observed from the pedestrian trajectory diagram. Target drift angle is further calculated and analyzed. Results indicate that target drift angle curve presents relatively large deviation to avoid pedestrian obstacle whilst not obvious deviation to avoid suitcase obstacle. Then, an interesting discovery shows that pedestrians tends to accelerate after passing obstacles no matter for the ascending or the descending process. Finally, a pedestrian obstacle benefits the ascending efficiency while slow down the descending process. The systematic experimental data can not only be used for the verification and validation of pedestrian models but also can provide a benchmark for the design of related facilities aiming at improving traffic efficiency.
\end{abstract}

Keywords Obstacle avoidance $\cdot$ unidirectional experiment · ascent and descent stairs 


\section{Introduction}

With the rapid development of modern cities, large public buildings have become more and more complex to accommodate the increasing population. In such buildings, pedestrians prefer to choose the shortest path to get to the exit and minimize movement or evacuation time when there are no obstacles inside the pedestrian facilities. However, once obstacles present, for the reason that those obstacles cannot be crossed over, pedestrians will detour to avoid potential collisions. As a consequence, the pedestrian's motion features and evacuation path will be influenced, resulting in complex path selection and evacuation efficiency problem in large public buildings. Stair is a critical pedestrian connecting different levels of a building, and serves as the only escape passageway under most scenarios. Therefore, it is essential to study the influence of obstacle pedestrian movement features on stairs.

Many scholars have studied the influence of obstacle by performing experimental and simulation research. Up to now, studies on the topic of exploring the effect of obstacle on pedestrian motion features mainly focus on the effects of obstacle size [1], position $[2,3]$, shape [4,5], spatial layout [6-8] and type [9] on evacuation efficiency and path selection features [10]. It has been found that obstacles influence pedestrian movement characteristics and evacuation efficiency [11]. Recently, Chen et al. [6] conducted an experimental study to investigate the effect of obstacle layouts on pedestrian flow features in corridors. Their study found that flow rates in the non-parallel layout was greater than that in the parallel layout. With a similar obstacle space layout, Li et al. [10] conducted field observations and a virtual experiment to study pedestrian route choice behavior around obstacles. The study found that pedestrians route choice depends on pedestrian density along evacuation routes, to be more specific, less used routes are preferred. Wang et al. [9] performed a series of controlled experiments to explore the influence of fixed obstacles and temporary obstacles on the unidirectional pedestrian flow in a corridor, interestingly, the study found that pedestrians would change their walking direction and reducing velocity at varied distances away from the fixed obstacle.

Summarizing the above studies, it can be found that the effects of obstacle on pedestrian movement characteristics and passing efficiency on level ground has been focused on. It should be noticed that stairs are typically three-dimensional structure and usually bottlenecks in built environment. The stair geometry features have impact on pedestrian movement characteristic. As a consequence, by performing a well-structured single-file pedestrian flow experiment, Chen et al. [12] firstly studied fundamental diagram of pedestrians descent and ascent stairs. Then, Wang et al. [13] found that stair gradient have effect on pedestrian density-specific flow relation. However, the impact of obstacle on obstacle avoidance characteristics on staircase has not been systematically studied yet. Therefore, a series of unidirectional pedestrian movement experiments under controlled laboratory conditions were conducted to study the effect of fixed obstacles on pedestrian movement characteristic on ascending and descending stair.

The rest of the paper is organized as follows. The experimental setup is introduced in Sec. 2. The pedestrian trajectory extraction and processing method is elaborated in Sec. 3. In Sec. 4, the target drift angle, speed of pedestrians and flow rates are discussed 
Table 1 Setup of the obstacle in each experimental run

\begin{tabular}{ccccc}
\hline & \multicolumn{2}{c}{ Obstacle position } & \multicolumn{2}{c}{ Type of obstacle } \\
& Ascending & Descending & Pedestrian & Suitcase \\
\hline S1 & $*$ & & $*$ & \\
S2 & $*$ & & & $*$ \\
S3 & & $*$ & $*$ & \\
S4 & & $*$ & & $*$ \\
\hline
\end{tabular}

and analyzed in detail. Sec. 5 presents the conclusions of the paper.

\section{Experimental setup}

A series of controlled experiment is performed on an experimental staircase in Southwest Jiaotong University in October 2020, including single-file luggage-laden experiment and unidirectional experiment of obstacles avoidance on stair, this paper mainly discussed the effect of obstacles on movement characteristic of pedestrians. The geometrical sketch of the staircase and a snapshot of the unidirectional descending and ascending experiment on the stair can be found in Fig. 1. The experimental staircase is divided into three region, i.e., ground landing area, flight and top landing area, as shown in Fig. 1(a) and Fig. 1(c). There are 10 steps connecting the top landing area and the ground landing area. The riser height and tread depth are $20 \mathrm{~cm}$ and $30 \mathrm{~cm}$, respectively. The slope of the stair is thus $34^{\circ}$. More detailed information about the experimental staircase can be found in [14]. In total 35 volunteers, who were all students from the Southwest Jiaotong University, were recruited to participate in this experiment. Their average age is 23.6 ranging from 19 to 28, while their average height is $166 \mathrm{~cm}$ ranging from 155 to $183 \mathrm{~cm}$.

Different from the movement on level plane [15-17], the movement on the stairs is a little complex and can be divided into three stages, namely, descending, level movement and ascending. Two types of obstacle were considered. The first type was a pedestrian obstacle where a standing still pedestrian was set as the fixed obstacle, as shown in Fig. 1(a). The second type was a suitcase obstacle, as shown in Fig. 1(c). The fixed obstacle was placed in the middle position of the flight area. In total 4 runs of experiments were designed and each run has been performed repeatedly for 5 times according to Tab. 1. Each run is mutually independent in ascending and descending process. The participants were allowed to use handrails and overtake surrounding pedestrians. Meanwhile, in order to reduce the effect of fatigue, the participants were ordered to rest in between the experimental runs.

A camera with a resolution of $1920 \times 1080$ was mounted $10 \mathrm{~m}$ above the ground to record the whole experimental process. For the convenient of extracting pedestrian trajectory, each participant was required to wear a red cap with a white dot in the center. The PeTrack software [18] was used to automatically extract the trajectory pixel coordinates from the experimental video. The coordinates of the pedestrians from image space can be 


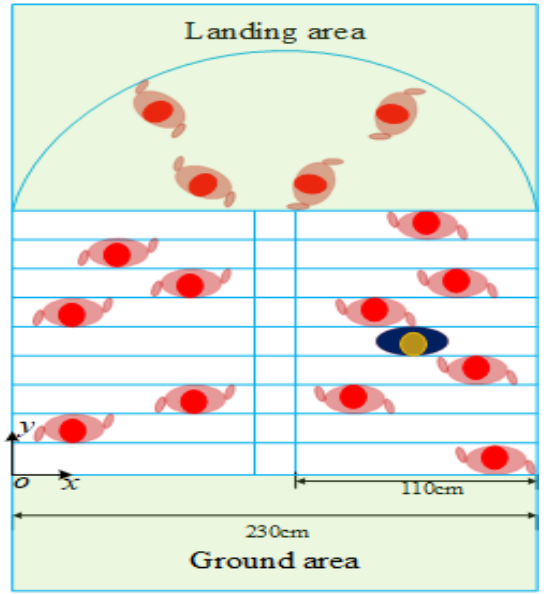

(a)

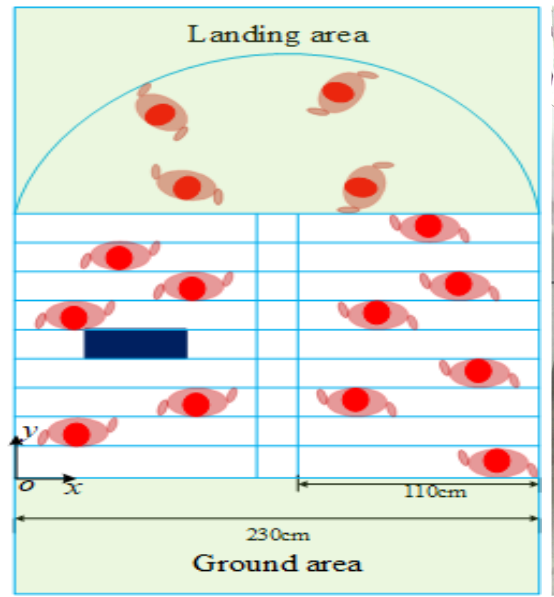

(c)

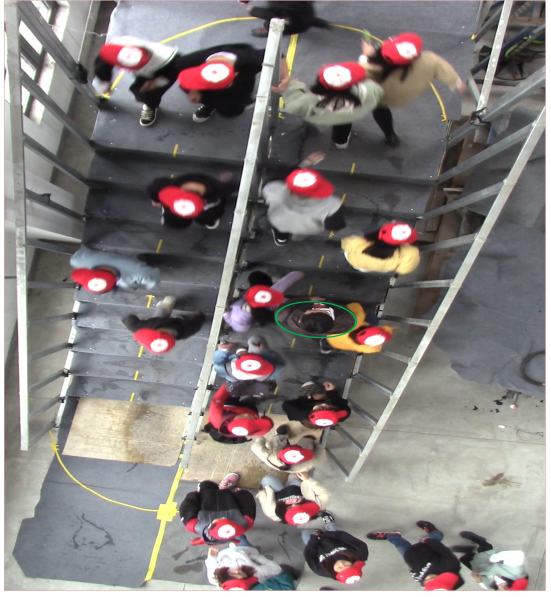

(b)

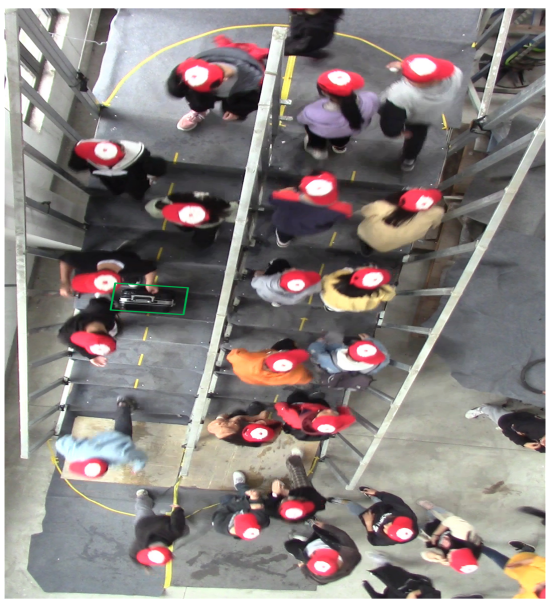

(d)

Figure 1 Illustration of experiment setup. (a) and (c) are sketches of the scenario, (b) and (d) present snapshots of the unidirectional experiment where the pedestrian obstacle is marked with a green oval while the suitcase obstacle is marked with a green rectangle.

transformed into real space using the direct linear transformation method [19]. Based on the above method, the actual physical coordinates of the pedestrian are obtained.

\section{Trajectory extraction and processing}

\subsection{Pedestrian trajectory extraction}

Due to the reason that pedestrian movement on stairs is complex and three-dimensional, for the convenient of processing, we further project the three-dimensional movement onto the horizontal plane. The two-dimension coordinate system is built in Fig. 1(a) and Fig. 1(c). Based on the row trajectory coordinates, the pedestrian trajectory diagram 
in different runs are shown in Fig. 2. Comparing scenarios S1 and S2, we can see that the pedestrians show clear reaction to pedestrian obstacle rather than suitcase obstacle. Similarly, in scenarios S3 and S4, similar features can be observed. Meanwhile, another phenomenon is also observed, as shown in Fig. 2(a) and Fig. 2(c), the avoidance behavior was performed at the position one step away from obstacle.

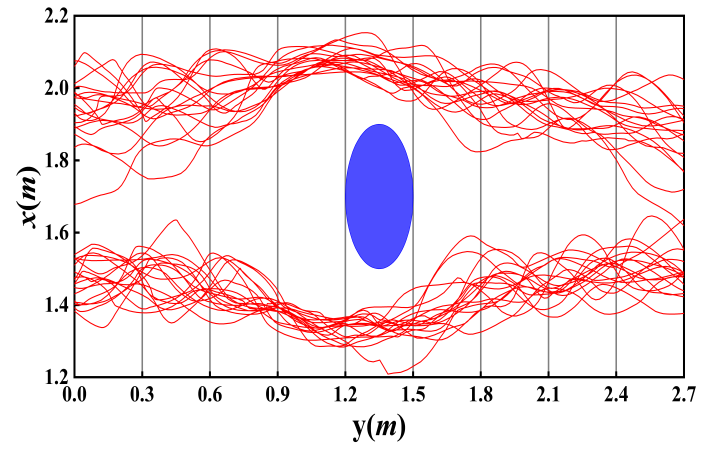

(a) S1

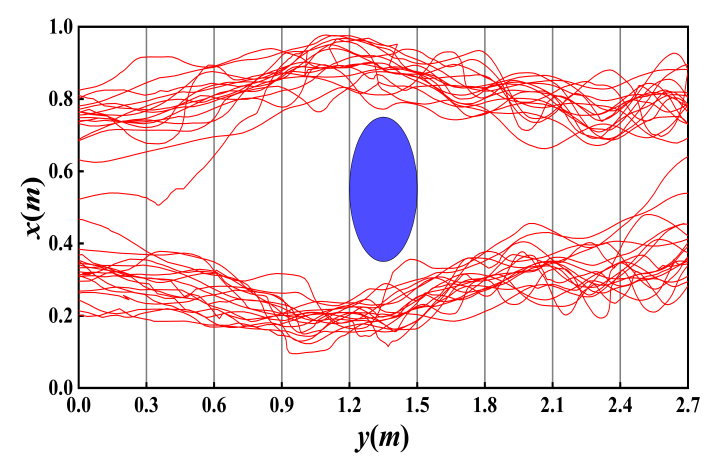

(c) S3

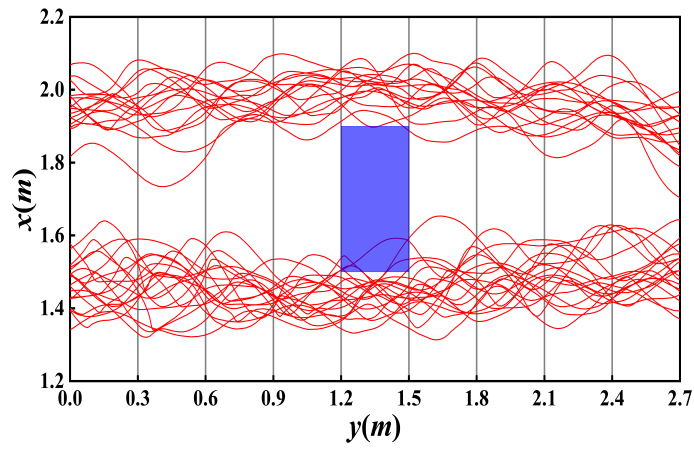

(b) $\mathrm{S} 2$

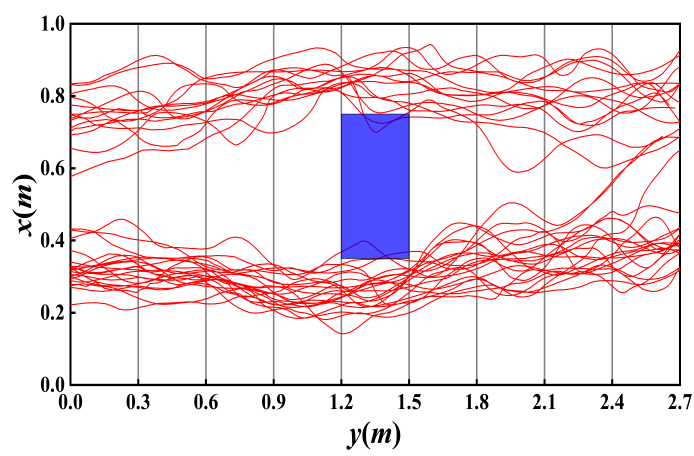

(d) S4

Figure 2 Pedestrian trajectories in different runs. The blue ellipse indicates the pedestrian obstacle. The blue rectangle represents the suitcase obstacle. The gray line indicates the step edge. S1 and S2 are ascending processes, S3 and S4 are descending processes.

\subsection{Data processing method}

The individual instantaneous velocity $v_{i}(t)$ is calculated by Eq. 1

$$
v_{i}(t)=\frac{\left\|\vec{r}_{i}\left(t+\frac{\Delta t}{2}\right)-\vec{r}_{i}\left(t-\frac{\Delta t}{2}\right)\right\|}{\Delta t}
$$

where $\vec{r}_{i}\left(t+\frac{\Delta t}{2}\right)$ is the position coordinates of the pedestrians $i$ at the time $t+\frac{\Delta t}{2}$, and the time interval is $0.4 \mathrm{~s}$.

The flow rate $f$ is calculated as follows Eq. 2 .

$$
f=\frac{N}{\Delta t}
$$


where $N$ is the total number of pedestrians in the experiment, and $\Delta t$ is the total time spent by all pedestrians passing through the nine steps on the stair.

Based on the shortest path principle, pedestrians try to select the straightway (shortest path) to get to their destinations in normal conditions. However, pedestrians have to change their movement direction to avoid collision when they facing obstacle in stair. Wang et al. [9] introduced target drift angle to analyze the variation of pedestrians trajectory in the corridor of horizontal plane. Similarly, we adopt the same method to analyze movement of pedestrians on the stair. As shown in Fig. 3, the target drift angle $\theta_{i}(t)$ is defined as the intersection angle between the straight line at target point which parallel to the $y$ axis and the moving direction of pedestrians $i$ at time $t$.

$$
\theta_{i}(t)=\arccos \frac{\left\|\vec{r}_{i}(t+\Delta t)-\vec{r}_{i}(t)\right\|^{2}+\left\|\vec{D}_{i}(t)-\vec{r}_{i}(t)\right\|^{2}+\left\|\vec{r}_{i}(t+\Delta t)-\vec{D}_{i}(t)\right\|^{2}}{2\left\|\vec{r}_{i}(t+\Delta t)-\vec{r}_{i}(t)\right\|\left\|\vec{D}_{i}(t)-\vec{r}_{i}(t)\right\|}
$$

where $\vec{r}_{i}(t+\Delta t)$ is the position coordinates of the pedestrians $i$ at the time $t+\Delta t, \vec{D}_{i}(t)$ indicates the position coordinates of the target point of pedestrian $i$ at time $t$, where the target point of ascending process is $\vec{D}_{i}(t)=\left(2.7, x_{i}(t)\right)$ and the target point of descending process is $\vec{D}_{i}(t)=\left(0, x_{i}(t)\right)$.

In order to analyze the evolution of position and target drift angle, the left deflection $\left(A_{i}(t)>0\right)$ and right deflection $\left(A_{i}(t)<0\right)$, along the $y$ coordinate is defined by comparing the position coordinate of the pedestrian at time $t$ and $t+\Delta t . A_{i}(t)$ of ascending process and descending process is calculated by Eq. 4 and Eq. 5, respectively.

$$
\begin{aligned}
& A_{i}(t)=\left\{\begin{array}{cc}
\theta_{i}(t), & x_{i}(t+\Delta t)<x_{i}(t) \\
-\theta_{i}(t), & x_{i}(t+\Delta t)>x_{i}(t)
\end{array}\right. \\
& A_{i}(t)=\left\{\begin{array}{cc}
-\theta_{i}(t), & x_{i}(t+\Delta t)<x_{i}(t) \\
\theta_{i}(t), & x_{i}(t+\Delta t)>x_{i}(t)
\end{array}\right.
\end{aligned}
$$

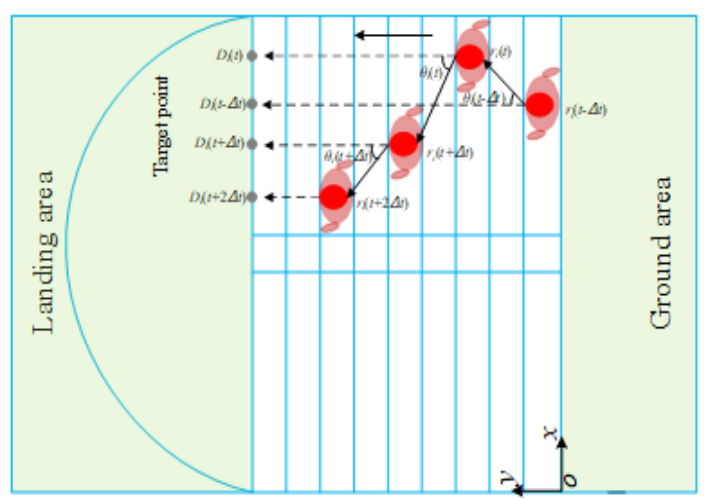

(a)

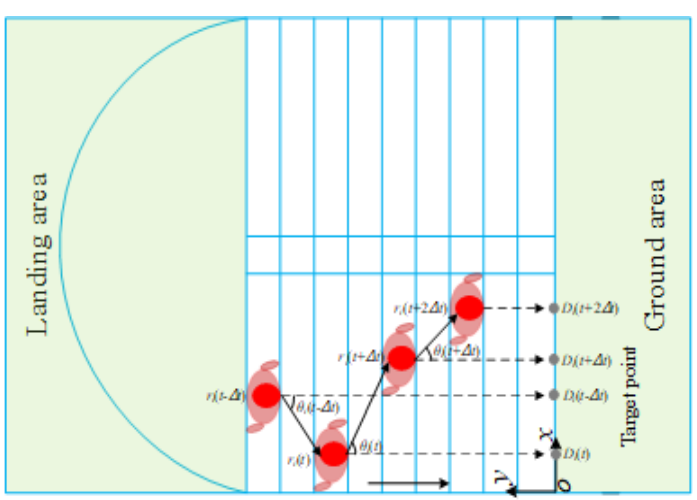

(b)

Figure 3 The schematic diagram of the target drift angle: (a) ascending process and (b) descending process. 


\section{Results and Discussions}

\subsection{Target drift angle}

In order to quantify the obstacle avoidance characteristics, the target drift angle has been calculated based on the trajectory coordinates. We estimated the mean values and standard deviations of target drift angle averaging over position and analyzed their mutual dependence. The evolution of position and target drift angle towards left and right along the $y$ coordinate is shown in Fig. 4. The variation of target drift angle towards left and right against direction of descending and ascending process can be observed in Fig. 4. It can be found that the target drift angle curve presents relatively large deviation to avoid pedestrian obstacle, as indicated by the lines in the green dotted circle. At the position $y=1.2 \mathrm{~m}$, a deviation can be observed in scenario S1, as show Fig. 4(a). However, the target drift angle curve at the position $y=1.5 \mathrm{~m}$ presents a smaller amplitude in scenario S3. The main reason is that the obstacle was placed in between $y=1.2 \mathrm{~m}$ and $y=1.5 \mathrm{~m}$, pedestrian have to change their walking direction at $y=1.2 \mathrm{~m}$ for ascending process, while pedestrian passed the obstacle at $y=1.5 \mathrm{~m}$ during descending process. Comparing the target drift angle curve of S1 and S2, it can be found that the deviation amplitude in scenario $\mathrm{S} 1$ is greater than that in scenario S2. That is to say, the type of obstacle has influence on pedestrian's avoidance characteristics. The target drift angle deviation during ascending process is lager the one during descending process. The findings is consistent with the pedestrian trajectory deviation in Fig. 2.

To further explore the influence of the obstacle on target drift angle, we calculate the average target drift angle towards left and right, respectively, and show the results in Fig. 5. For the ascending process, the average target drift angle towards left in scenario $\mathrm{S} 1$ is $17.04^{\circ}$, the average target drift angle towards right in scenario $\mathrm{S} 1$ is $-16.9^{\circ}$, however, the average target drift angle towards left and right in scenario S2 are both $29.4 \%$ and $23.7 \%$ less than that of scenario S1. To examine the statistical significance of obstacle type on target drift angle, parametric pairwise hypothesis test was conducted. The results indicated that the difference was significant between scenario S1 and S2 in Fig. 5. Similarly, the average target drift angle towards left and right in scenario S4 are both $29 \%$ and $27.5 \%$ less than scenario S3 for descending process. Considering that the height and shape of the pedestrian obstacle are both greater than that of the suitcase obstacle, the pedestrians will adopt collision avoidance in advance, resulting in a smaller target drift angle. To further compare the ascending and descending process, we tested target drift angle difference and found the difference was significant. The above result is in consistent with the findings from the Fig. 4.

We compared the target drift angle for pedestrian movement on horizontal plane and stair. As shown in Fig. 2(b) and Fig. 2(d), pedestrian trajectory show basically the same feature with normal walking, therefore, scenario S2 and S4 were regarded as normal walking on stair. Wang et al. [9] found that the average target drift angle towards left and right was about $7.2^{\circ}$ and $-7.4^{\circ}$ on horizontal flat, respectively. However, this study indicated that average target drift angle towards left and right was about $13.16^{\circ}$ and $-13.66^{\circ}$ in the ascending process, and about $12.46^{\circ}$ and $-12.42^{\circ}$ in the descending process. Considering 


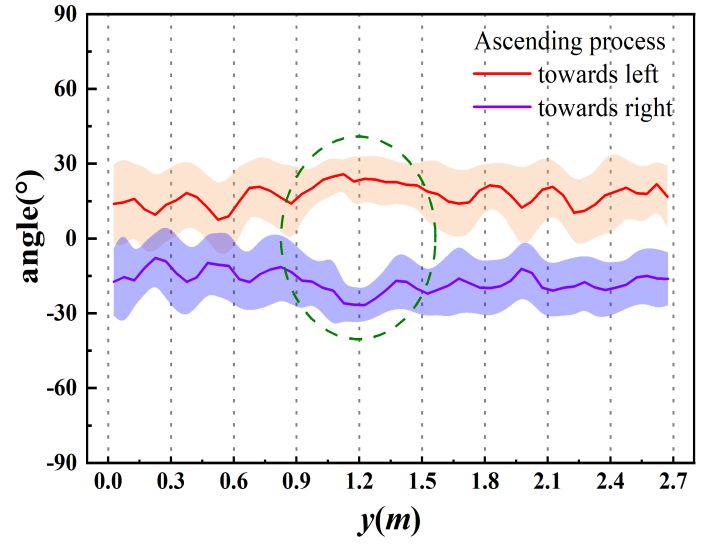

(a) $\mathrm{S} 1$

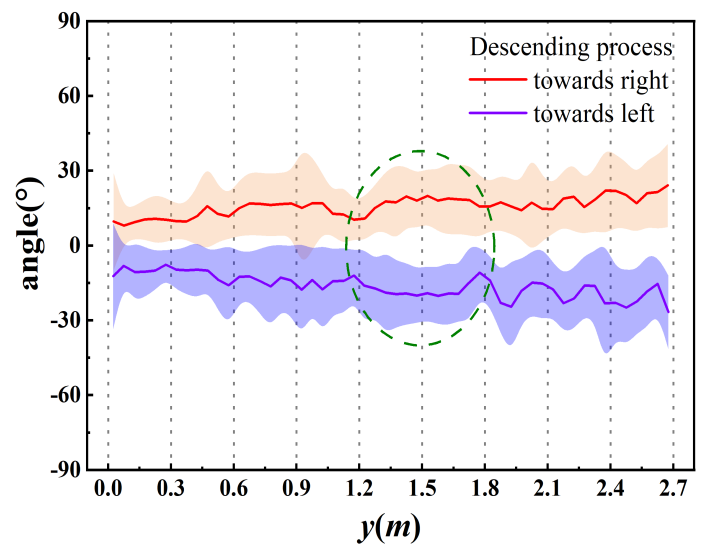

(c) $\mathrm{S} 3$

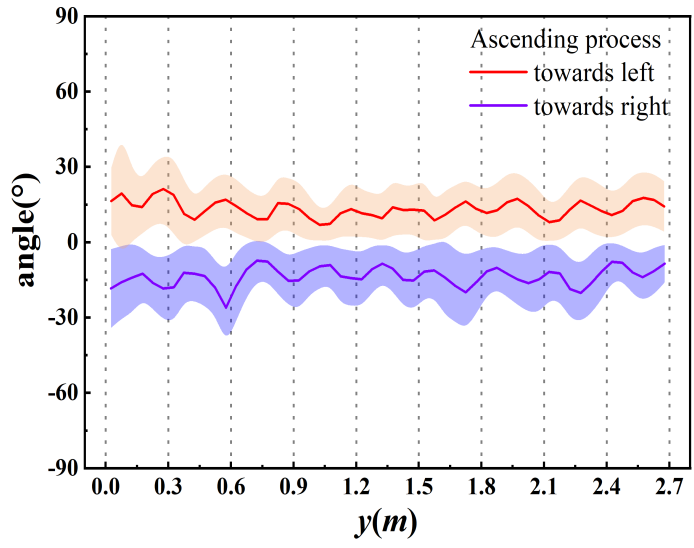

(b) S2

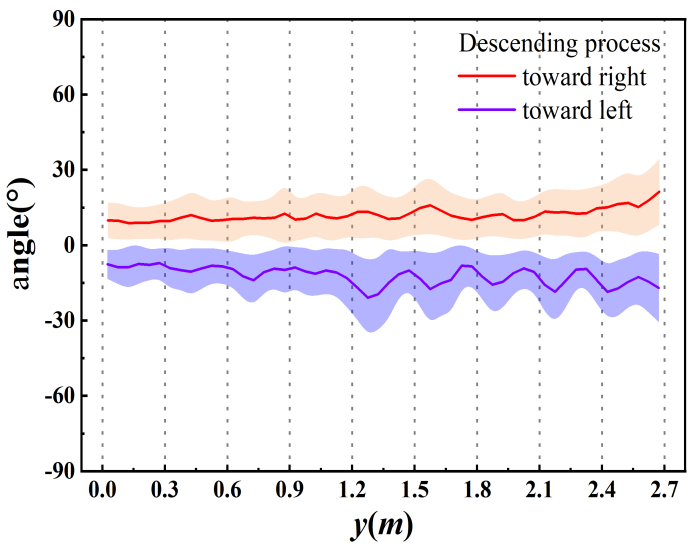

(d) $\mathrm{S} 4$

Figure 4 Evolution of position and target drift angle toward left and right along the $y$ coordinate. The dot line indicates the number of steps. The curve is obtained by the mean value for pedestrian position over a segment of $0.05 \mathrm{~m}$ and the shaded area represents the standard deviation.

that pedestrian lateral sway is lager on stairs, these larger angle values basically reflected the natural oscillation amplitude of a pedestrian.

In addition, Wang et al. [9] found most obvious change of the target drift angle curve at the position before the obstacle, however, the target drift angle curve at the position before the obstacle smoothly fluctuates on stair in Fig. 4. The amplitude towards left or right is about $25.8^{\circ}$ and $-26.5^{\circ}$ in ascending process and is about $19.6^{\circ}$ towards left and $-20.7^{\circ}$ towards right in descending process. The main reason is that the obstacle avoidance on stair resulted in larger target drift angle deviation. Interestingly, another microscopic pedestrian movement characteristic is observed, i.e., after passing by the obstacle, some pedestrian return to their original route in Fig. 4(a), while others choose the route which deviated a little bit from his/her original one, as shown in Fig. 4(c). 


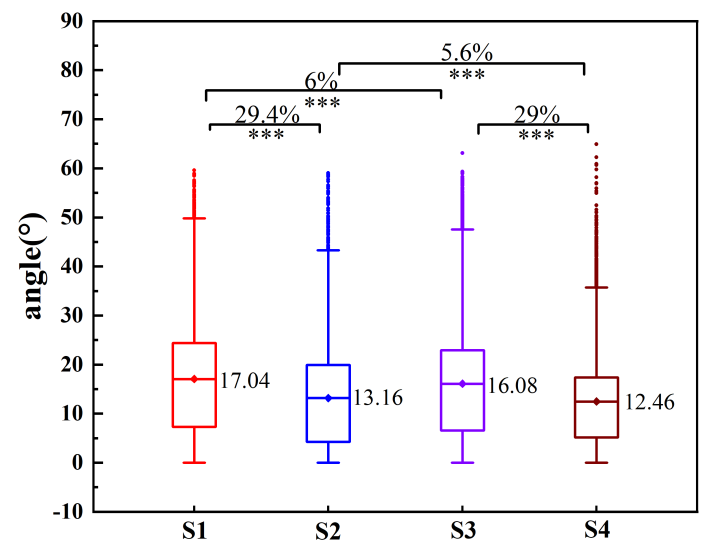

(a) Towards left

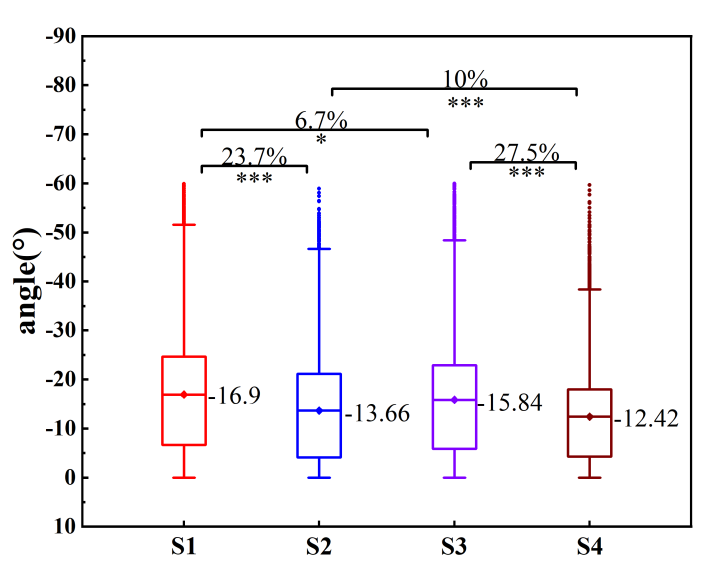

(b) Towards right

Figure 5 The box-and-whisker plots for the 25th and 75th percentiles of mean target drift angle. The numbers beside the box indicate the corresponding mean values. The connecting lines show the between group difference. The $p$-values are indicated as asterisks $\left(* p<0.05\right.$ and $\left.{ }^{* * *} p<0.001\right)$

\subsection{Speed of pedestrian}

The target drift angle only reflected avoidance characteristic in the direction, we further discuss the influence of the obstacle on the speed of pedestrians. Evolution of position and speed along the $y$ coordinate in the ascending process and descending process is plotted in Fig. 6. The vertical binning procedure was adopted, whole data region is divided into a series of bins per $0.05 \mathrm{~m}$, then the number of speed falling into each bin was counted and the mean value and standard error were calculated. In ascending process, the mean speed of scenarios S1 and S2 before passing obstacle is basically the same, however, the mean speed of scenario S1 is greater than that of scenario S2 after passing obstacles. On the contrary, the mean speed of scenario S4 is greater than that of scenario S3 before passing obstacles, the mean speed of scenario S3 and scenario S4 is basically the same after passing obstacles. This indicated that obstacle has a certain impact on spatial distribution of speed. Interestingly, it was observed that the pedestrians start to accelerate after passing obstacles no matter for the ascending process (except for $\mathrm{S} 2$ ) or the descending process. The slowing down phenomenon before the obstacle could be due to the local density increasing, however, we found no clear jam in front of the obstacle in the video recordings.

The average speed in different scenarios on staircase is further discussed by calculating the ascending and descending speed, as shown in Fig. 7. For the ascending process, the average speed of scenario S2 is $4.7 \%$ less than that of scenario S1, indicating the pedestrian obstacle motivated the movement of other pedestrians. Inversely, the average speed of scenario S3 is $7.9 \%$ less than that of scenario S4 in descending process, which is opposite to that of ascending process. This finding has also observed in the evolution of position and speed, as shown in Fig. 6. In terms of the difference between ascending process and descending process, which is caused by combined effect of the stair structure and the obstacle. In order to discuss difference of speed before and after obstacle, the average speed of ascending and descending process is plotted in Fig. 8. From the result it 


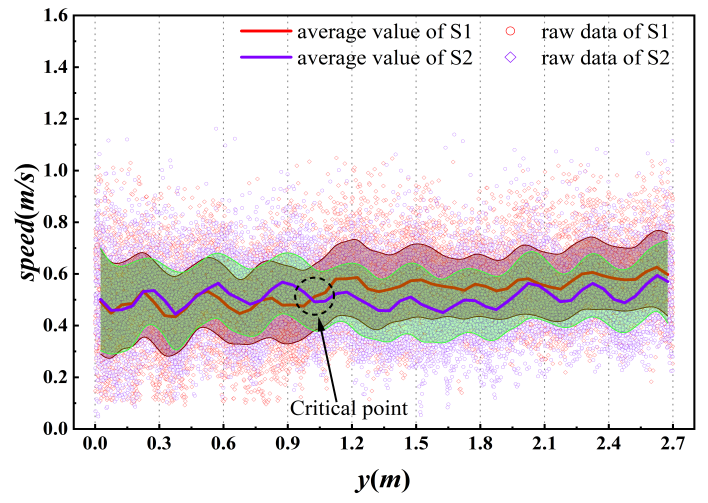

(a) Ascending process

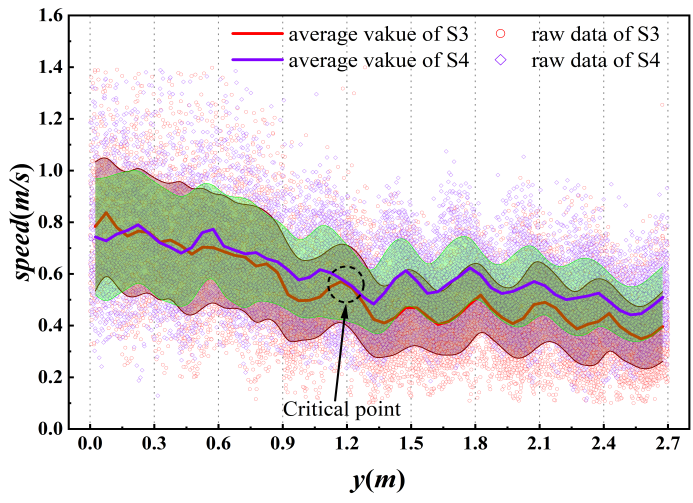

(b) Descending process

Figure 6 Evolution of position and speed along the $y$ coordinate in the ascending process and descending process. The dot line indicate the number of step. The curve is obtained by the mean value for position over a segment of $0.05 \mathrm{~m}$ and the shaded area represents the standard deviation.

can also be found that the average speed after passing obstacle is greater than that before passing obstacle, which is consistent with observed phenomenon in Fig. 6.

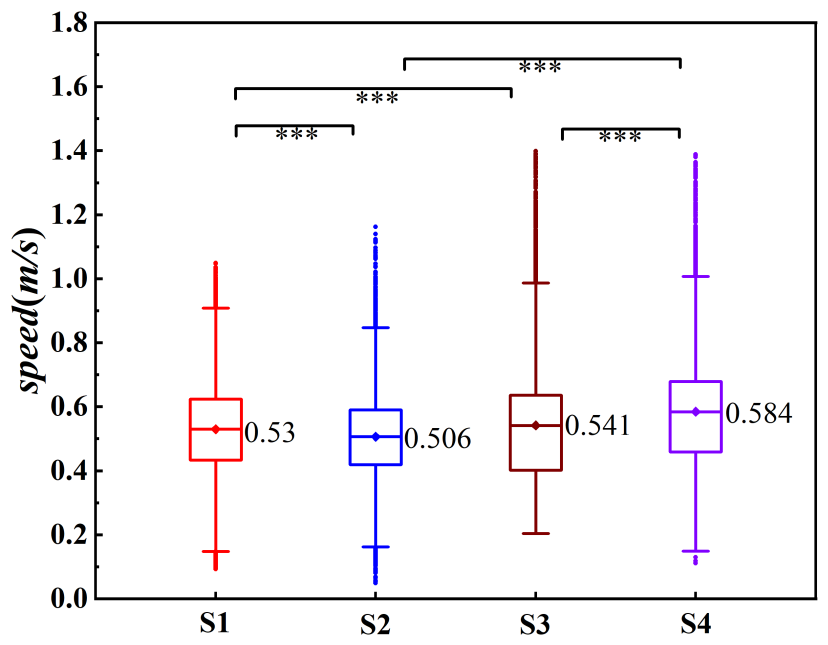

Figure 7 The box-and-whisker plots for the 25 th and 75 th percentiles of mean speed. The numbers beside the box indicate the corresponding mean values. The connecting lines show the between group difference. The $p$-values are indicated as asterisks $\left(* p<0.05\right.$ and $\left.^{* * *} p<0.001\right)$.

\subsection{Flow rates}

Flow rates in unidirectional flow on the staircase is shown in Fig. 9. It is no difficult to find that the flow rates of scenario S1 is 5.9\% higher than that of scenario S2 for ascending process. However, for descending process, flow rates of scenario S3 is $18 \%$ less than that of scenario S4. This indicates that the pedestrian obstacle accelerated the flow efficiency in 


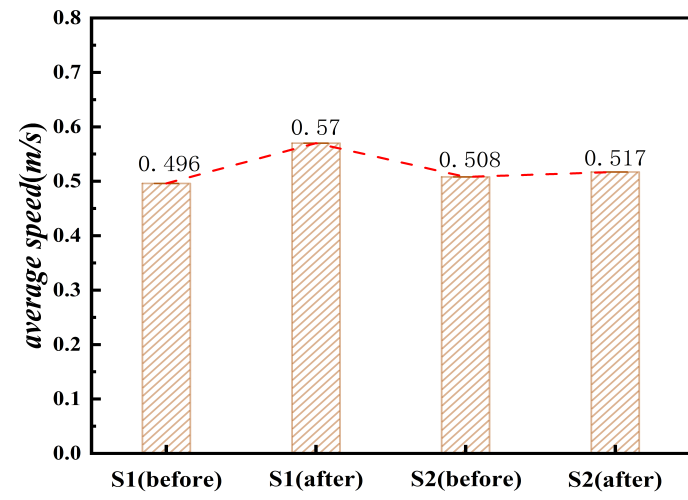

(a) Ascending process

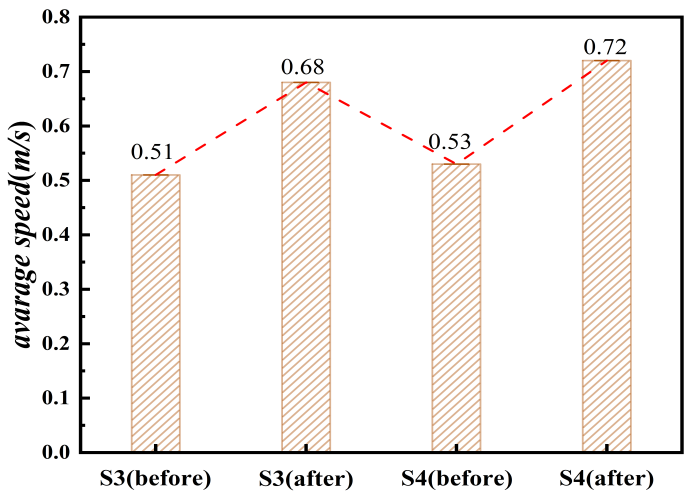

(b) Descending process

Figure 8 The average speed between before and after obstacles for different experimental scenarios.

ascending process, while on the contrary impede the movement of pedestrians in descending process. This finding is in consistent with the result of speed in Sec. 4.2. Meanwhile, the flow rates of scenario S4 is about 1.5 times than that of scenario S2, however, the flow rates of scenario $\mathrm{S} 3$ is about 1.3 times than that of scenario $\mathrm{S} 1$. The difference may due to that the pedestrian obstacle occupied a greater space when compared with the suitcase obstacle. From this point of view, the effect of obstacle type on pedestrian flow rates on stairs still needs further investigation.

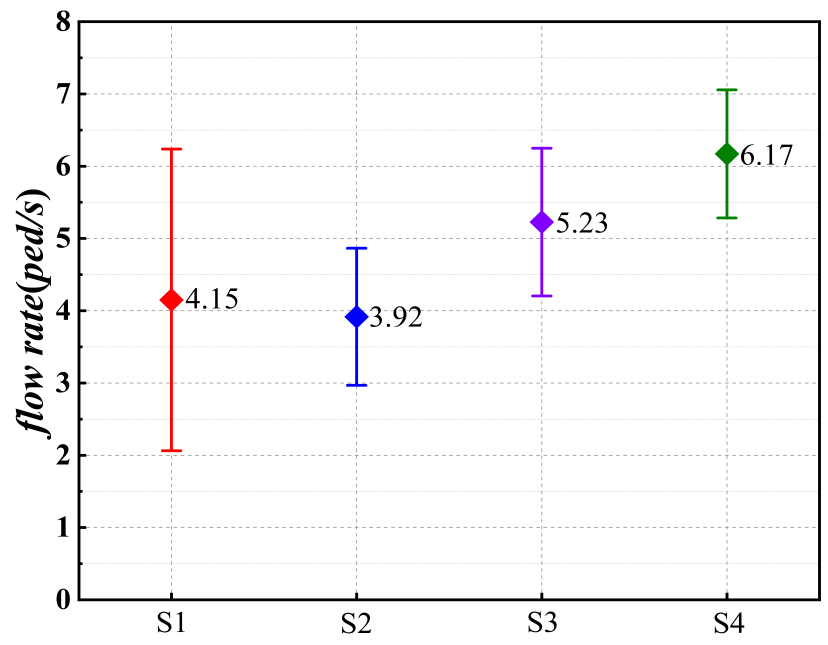

Figure 9 Flow rate in unidirectional flow on stair. The error bars represent the standard deviation.

\section{Conclusions}

To study the effect of fixed obstacles on pedestrian movement characteristic on stair, a series of unidirectional pedestrian movement experiments under controlled laboratory conditions were designed. Pedestrian trajectory has been extracted, from which the obstacle 
avoidance behavior is observed, i.e., pedestrian start to react at the position one step away from the obstacle. In order to quantify the avoidance characteristics on stairs, target drift angle towards left and right was calculated and analyzed. Firstly, target drift angle curve presents relatively large deviations to avoid pedestrian obstacle, then the target drift angle curve fluctuation is observed at $y=1.2 \mathrm{~m}$ for scenario $\mathrm{S} 1$ and $y=1.5 \mathrm{~m}$ for scenario S3. Secondly, the average target drift angle towards left and right of scenario S2 both are $29.4 \%$ and $23.7 \%$ less than that of scenario S1, respectively. The curve trend of scenarios S3 and S4 is consistent with above result. Thirdly, the movement characteristic difference between horizontal plane and stair were discussed. It was found that target drift angle on stair was greater than that on level flat. What is more, the pedestrians accelerated after passing obstacles no matter for the ascending or descending process. From a quantitative perspective, the average speed of scenario S3 is $7.9 \%$ less than that of scenario S4 in descending process, this result is contrary to that of ascending process. Finally, flow rates in unidirectional flow on the staircase was analyzed, the study indicated that the pedestrian obstacle could benefit the flow efficiency in ascending process while impede the movement of pedestrians in descending process.

Although the influence of fixed obstacles on the movement characteristic of pedestrians on stairs has been discussed, it should be admitted that the experimental scenarios were relatively simple, in the future, more experiments considering factors including obstacle size would be performed.

\section{Acknowledgement}

The authors deeply acknowledge the supports from the National Natural Science Foundation of China (Nos. 71871189, 72104205), the Science and Technology Development Funds of Sichuan Province (No. 2020YFS0291), the Research Project from the China Railway Eryuan Engineering Group Co. Ltd KYY2019086(19-20).

\section{References}

[1] Feliciani, C., Zuriguel, I., Garcimartin, A., Maza, D., Nishinari, K.: Systematic experimental investigation of the obstacle effect during non-competitive and extremely competitive evacuations. Scientific reports 10(1), 15947 (2020)

[2] Shi, X., Ye, Z., Shiwakoti, N., Tang, D., Lin, J.: Examining effect of architectural adjustment on pedestrian crowd flow at bottleneck. Physica A: Statistical Mechanics and its Applications 522, 350-364 (2019)

[3] Yanagisawa, D., Kimura, A., Tomoeda, A., Nishi, R., Y., S., Ohtsuka, K.: Introduction of frictional and turning function for pedestrian outflow with an obstacle. Phys Rev E 80, 036110 (2009) 
[4] Zhao, Y., Li, M., Lu, X., Tian, L., Yu, Z., Huang, K., Wang, Y., Li, T.: Optimal layout design of obstacles for panic evacuation using differential evolution. Physica A: Statistical Mechanics and its Applications 465, 175-194 (2017)

[5] Jia, X., Yue, H., Tian, X., Yin, H.: Simulation of pedestrian flow with evading and surpassing behavior in a walking passageway. Simulation 93(12), 1013-1035 (2017)

[6] Chen, S., Fu, L., Fang, J., Yang, P.: The effect of obstacle layouts on pedestrian flow in corridors: An experimental study. Physica A: Statistical Mechanics and its Applications 534, 122333 (2019)

[7] Lin, P., Ma, J., Liu, T., Ran, T., Si, Y., Wu, F., Wang, G.: An experimental study of the impact of an obstacle on the escape efficiency by using mice under high competition. Physica A: Statistical Mechanics and its Applications 482, 228-242 (2017)

[8] Oven, V.A., Cakici, N.: Simulation of the influence of spatial obstacles on evacuation pedestrian flow in walking facilities. Physica A: Statistical Mechanics and its Applications 571, 125844 (2021)

[9] Wang, W., Zhang, J., Li, H., Xie, Q.: Experimental study on unidirectional pedestrian flows in a corridor with a fixed obstacle and a temporary obstacle. Physica A: Statistical Mechanics and its Applications 560, 125188 (2020)

[10] Li, H., Zhang, J., Xia, L., Song, W., Bode, N.: Comparing the route-choice behavior of pedestrians around obstacles in a virtual experiment and a field study. Transportation Research Part C: Emerging Technologies 107, 120-136 (2019)

[11] Jia, X., Feliciani, C., Yanagisawa, D., Nishinari, K.: Experimental study on the evading behavior of individual pedestrians when confronting with an obstacle in a corridor. Physica A: Statistical Mechanics and its Applications 531, 121735 (2019)

[12] Chen, J., Lo, S., Ma, J.: Pedestrian ascent and descent fundamental diagram on stairway. Journal of Statistical Mechanics: Theory and Experiment 2017, 083403 (2017)

[13] Wang, J., Ma, J., Lin, P., Sarvi, M., Li, R.: Pedestrian single file movement on stairway: Investigating the impact of stair configuration on pedestrian ascent and descent fundamental diagram. Safety Science 143, 105409 (2021)

[14] Shi, D., Ma, J., Luo, Q., Li, X., Chen, J., Lin, P.: Fundamental diagrams of luggageladen pedestrians ascending and descending stairs. Physica A: Statistical Mechanics and its Applications 572, 125880 (2021)

[15] Ma, J., Shi, D., Li, T., Li, X., Xu, T., Lin, P.: Experimental study of single-file pedestrian movement with height constraints. Journal of Statistical Mechanics: Theory and Experiment 2020(7), 073409 (2020) 
[16] Ma, J., Shi, D., Li, T.: Pedestrian fundamental diagram in between normal walk and crawling. Traffic and Granular Flow 2019 Springer Proceedings in Physics2020, 185-94 (2020)

[17] Zhang, J., Klingsch, W., Schadschneider, A., Seyfried, A.: Transitions in pedestrian fundamental diagrams of straight corridors and t-junctions. Journal of Statistical Mechanics: Theory and Experiment 2011(6), P06004 (2011)

[18] Boltes, M., Seyfried, A.: Collecting pedestrian trajectories. Neurocomputing 100, 127-33 (2013)

[19] Ma, J., Song, W., Fang, Z., Lo, S., Liao, G.: Experimental study on microscopic moving characteristics of pedestrians in built corridor based on digital image processing. Building and Environment 45(10), 2160-2169 (2010) 